\title{
A novel homozygous missense variant in MATN3 causes spondylo- epimetaphyseal dysplasia Matrilin 3 type in a consanguineous family
}

\section{Yasin, Samina}

2020-08

Yasin , S , Mustafa , S , Ayesha, A , Latif , M , Hassan , M , Faisal , M , Mäkitie , O , lqbal , F \& Naz , S 2020 , ' A novel homozygous missense variant in MATN3 causes spondyloepimetaphyseal dysplasia Matrilin 3 type in a consanguineous family ' , European Journal of Medical Genetics , vol. 63 , no. 8 , 103958 . https://doi.org/10.1016/j.ejmg.2020.103958

http://hdl.handle.net/10138/330653

https://doi.org/10.1016/j.ejmg.2020.103958

cc_by_nc_nd

acceptedVersion

Downloaded from Helda, University of Helsinki institutional repository.

This is an electronic reprint of the original article.

This reprint may differ from the original in pagination and typographic detail.

Please cite the original version. 


\section{Journal Pre-proof}

A novel homozygous missense variant in MATN3 causes spondylo-epimetaphyseal dysplasia Matrilin 3 type in a consanguineous family

Samina Yasin, Saima Mustafa, Arzoo Ayesha, Muhammad Latif, Mubashir Hassan, Muhammad Faisal, Outi Makitie, Furhan Iqbal, Sadaf Naz

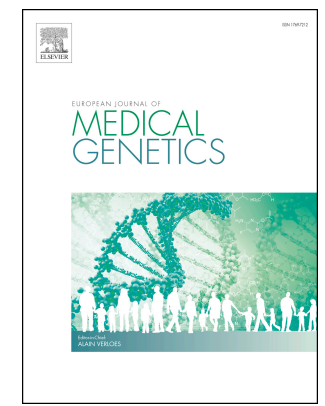

PII:

S1769-7212(20)30038-0

DOI:

https://doi.org/10.1016/j.ejmg.2020.103958

Reference: $\quad$ EJMG 103958

To appear in: European Journal of Medical Genetics

Received Date: 22 January 2020

Revised Date: 11 May 2020

Accepted Date: 17 May 2020

Please cite this article as: S. Yasin, S. Mustafa, A. Ayesha, M. Latif, M. Hassan, M. Faisal, O. Makitie, F. Iqbal, S. Naz, A novel homozygous missense variant in MATN3 causes spondylo-epimetaphyseal dysplasia Matrilin 3 type in a consanguineous family, European Journal of Medical Genetics (2020), doi: https://doi.org/10.1016/j.ejmg.2020.103958.

This is a PDF file of an article that has undergone enhancements after acceptance, such as the addition of a cover page and metadata, and formatting for readability, but it is not yet the definitive version of record. This version will undergo additional copyediting, typesetting and review before it is published in its final form, but we are providing this version to give early visibility of the article. Please note that, during the production process, errors may be discovered which could affect the content, and all legal disclaimers that apply to the journal pertain.

(C) 2020 Published by Elsevier Masson SAS. 


\section{Authorship statement}

Samina Yasin: Methodology, Investigation, Formal analysis, Data curation, Writing- Original draft preparation

Saima Mustafa: Methodology, Writing- Original draft preparation

Aarzoo Ayesha and Muhammad Latif: Methodology, Data curation, Final review of manuscript

Mubashir Hassan and Muhammad Faisal: Methodology, Formal analysis, Final review of manuscript

Outi Makitie: Writing- Clinical Reviewing

Furhan Iqbal: Conceptualization, Supervision, Final review of manuscript

Sadaf Naz: Conceptualization, Project administration, Supervision, Writing- Reviewing and Editing 


\section{A novel homozygous missense variant in MATN3 causes Spondylo-}

\section{epimetaphyseal dysplasia Matrilin 3 type in a consanguineous family}

Samina Yasin ${ }^{\# 1}$, Saima Mustafa ${ }^{\# 2}$, Arzoo Ayesha², Muhammad Latif ${ }^{3}$, Mubashir Hassan ${ }^{4}$, Muhammad Faisal $^{5}$, Outi Makitie ${ }^{6,7}$, Furhan Iqbal $^{2 \# *}$, Sadaf Naz $^{1 \# *}$

${ }^{1}$ School of Biological Sciences, University of the Punjab, Quaid-i-Azam Campus, Lahore, Pakistan

${ }^{2}$ Institute of Pure and Applied Biology, Zoology Division, Bahauddin Zakariya University Multan, Pakistan

${ }^{3}$ Department of Zoology, Division of Science and Technology, University of Education Lahore, Multan Campus, Multan, Pakistan

${ }^{4}$ Institute of Molecular Biology and Biotechnology (IMBB), University of Lahore, Pakistan ${ }^{5}$ Faculty of Health Studies, University of Bradford, United Kingdom

${ }^{6}$ Children's Hospital, University of Helsinki, Finland

${ }^{7}$ Folkhälsan Institute of Genetics, Helsinki, Finland

\#SM and SY equal first authors, \#FI and SN equal contribution

\section{*Corresponding authors}

Dr. Furhan Iqbal Institute of Pure and Applied Biology, Zoology Division. Bahauddin Zakariya University Multan, 60800, Pakistan, Phone: 923315657685, email:

furhan.iqbal@bzu.edu.pk

Dr. Sadaf Naz, School of Biological Sciences, University of the Punjab, Quaid-i-Azam

Campus, Lahore, Phone: 92429931819, email: naz.sbs@pu.edu.pk 


\section{Abstract}

2 Spondylo-epimetaphyseal dysplasia Matrilin 3 type (SEMD) is a rare autosomal recessive

3 skeletal dysplasia characterized by short stature, abnormalities in the vertebral bodies and long

4 bones, especially the lower limbs. We enrolled a consanguineous family from Pakistan in which

5 multiple siblings suffered from severe skeletal dysplasia. The six affected subjects ranged in

6 heights from 100 to $136 \mathrm{~cm}$ ( -6 standard deviation). Lower limb abnormalities with variable

7 varus and valgus deformities and joint dysplasia were predominant features of the clinical

8 presentation. Whole exome sequencing (WES) followed by Sanger sequencing identified a

9 missense variant, c.542G>A, p.(Arg181Gln) in MATN3 as the genetic cause of the disorder. The

10 variant was homozygous in all affected individuals while the obligate carriers had normal heights

11 with no skeletal symptoms, consistent with a recessive pattern of inheritance. Multiple sequence

12 alignment revealed that MATN3 domain affected by the variant is highly conserved in orthologous proteins. The c.542G>A, p.(Arg181Gln) variant is only the fourth variant in MATN3 causing an autosomal recessive disorder and thus expands the genotypic spectrum.

Key words: Exome sequencing; Pakistan; SEMD; Short stature; Skeletal dysplasia 


\section{Introduction}

Spondylo-epimetaphyseal dysplasia (SEMD) are a rare heterogeneous group of disorders including more than twenty different conditions distinguished by a combination of clinical, radiological and molecular characteristics and have different modes of inheritance (CormierDaire, 2008; Anthony et al., 2015). Skeletal abnormalities are characterized by spinal, epiphyseal and metaphyseal anomalies. Variants in genes encoding Cartilage Oligomeric Matrix Protein (COMP), Collagen Type 2 Alpha 1 (COL2A1), Perlecan, 3'-Phosphoadenosine 5'Phosphosulfate Synthase 2 (PAPSS2), Transcription Initiation Factor Kinase (EIFKA3), Matrilin-3 (MATN3) and Matrix Associated Actin-dependent Regulator of Chromatin (SMARCAL1) cause SEMD (Borochowitz et al., 2004; Jackson et al., 2012a; Mortier et al., 2019).

A rare autosomal recessive form of SEMD is Spondylo-epimetaphyseal dysplasia Matrilin 3 type. It is associated with abnormalities in the vertebral bodies and long bones, especially in the lower limbs; the changes involving both epiphyses and metaphyses. It is caused by biallelic variants in MATN3 (OMIM\# 602109) (Borochowitz et al., 2004). MATN3 is located on chromosome 2 and consists of eight exons (Belluoccio et al., 1998). It encodes a modular protein Matrilin-3 which is found specifically in the extracellular matrix of cartilage (Wagener et al., 2000). It has a Willebrand Factor A-domain, four epidermal growth factor (EGF) repeats and a coiled-coil carboxy terminal domain for oligomerization. MATN3 can make homotetramers or heterotetramers with Matrilin-1. MATN3 also has a high affinity to bind with both COMP and type IX collagen which are two important extracellular matrix components in cartilage (Cotterill et al., 2005; Bell et al., 2012). 
In the present study we used a whole exome sequencing approach to identify the causative variant of MATN3 in a consanguineous family with multiple individuals affected by severe skeletal dysplasia.

\section{Methods}

\section{Family recruitment and sample collection}

We recruited a Pakistani family PK-ST-KP-08 from Southern Punjab with multiple individuals affected with short stature and severe skeletal malformations. The ethical committee at Bahauddin Zakariya University, Multan and the IRB of School of Biological Sciences, University of the Punjab, Lahore approved this research. Written informed consents were obtained for all participants. Clinical data and radiographs were acquired. The full heights, the trunk sizes without the head sizes, the size of lower segment from hip bone to feet while standing and arm spans were measured for the affected individuals. Upper segment to lower segment (US/LS) ratios and arm span to height (AS/Height) ratios were calculated. Blood samples were collected from one unaffected and six affected individuals. Genomic DNA was extracted from whole blood using sucrose lysis and salting out.

\section{Whole exome sequencing and data analysis}

Whole exome sequencing (WES) was completed on genomic DNA of proband V:18 (Macrogen inc, South Korea). The data was analyzed using wANNOVAR (http:/wannovar.usc.edu/index.php) to annotate the variants. Exonic and splice site variants with an allele frequency of less than 0.01 were retained. Missense variants were prioritized on the basis of in silico predictions from Sorting Intolerant From Tolerant (SIFT) (http://sift.jcvi.org/), PolyPhen-2 (http://genetics.bwh.harvard.edu/pph2/), Protein Variation Effect Analyzer 
(PROVEAN) (http://provean.jcvi.org/index.php), Mutation Assessor

(http://mutationassessor.org/), Mutation Taster 2 (http://www.mutationtaster.org/), Rare Exome Variant Ensemble Learner (REVEL) and ClinPred (https://bio.tools/ClinPred). Conservation of amino acids was checked using UCSC genome browser (https://genome.ucsc.edu/) and homologene (http://www.ncbi.nlm.nih.gov/homologene). Comparison between the human protein sequence of MATN3 and that of five most diverse vertebrate species, mouse, chicken, alligator, frog and zebrafish was completed by obtaining their sequences from Uniprot (https://www.uniprot.org/) and alignment with Clustal Omega (https://www.ebi.ac.uk/Tools/msa/clustalo/). Associated phenotypes of genes affected by variants were accessed from Online Mendelian Inheritance in Man (OMIM) (https://www.omim.org/).

\section{Sanger sequencing}

Candidate variant was validated on DNA of all available family members by Sanger sequencing. Primers were designed using Primer 3 (http://bioinfo.ut.ee/primer3-0.4.0/) based on the genomic sequence of MATN3 (NM_002381.5) to amplify the exon 2 region containing the candidate variant. PCR products were sequenced using Big Dye Terminator v.3.1 (ABI Thermo Fisher) on ABI 3730. The shortlisted variant allele frequency was checked in 50 ethnically matched controls and all public databases.

\section{Model building of proteins}

The three dimensional (3D) structures of wild-type and mutant MATN3 were generated computationally by obtaining amino acids sequences from Uniprot Knowledge Database. A homology modelling approach was employed to predict 3D structures of wild-type and mutant MATN3. The automated Swiss modeller (https://swissmodel.expasy.org/) was employed to 
predict MATN3 structure. Two templates (1X6V \& 1JDN) having sequence identity (78.20 and $31.04 \%$, respectively) were selected to build the models.

\section{Results}

\section{Clinical characterization of patients}

The consanguineous family PK-ST-KP-08 included six affected individuals ranging in age from 15 to 45 years (Figure 1A). All affected individuals had short stature (100 cm to $136 \mathrm{~cm} \mathrm{Z-scores}$ $-6.3--6.8$ ), bowed legs and waddling gait (Figure 1B). Lower limb deformities predominated and varied from "windswept deformity" to varus or valgus deformity with flexion contractures at the knees (Figure 1B). No facial dysmorphism was observed. Radiographs of patients $1 \mathrm{~V}: 4, \mathrm{~V}: 7$ and V:8 (Figures 1C and 1D) revealed severe joint dysplasia both at the knees and hips with abnormal metaphyseal shape at the distal femurs and femoral neck valgus or varus deformity. Femoral neck was short and the articular space reduced. The length of lower limb long bones was significantly reduced. Affected individuals IV:4, IV:6, IV:7, IV:8, V:7 and V:8 had heights of $123,115,136,131,130$ and $100 \mathrm{~cm}$ with around -6.0 standard deviations (SD) for all. Their trunk sizes were $46,51,43,44,43$ and $45 \mathrm{~cm}$, ranging from -2.0 to -6.0 SD while the arm spans were $152,157,160,150,135$ and $136 \mathrm{~cm}$. Trunk size to lower segment ratios ranged from 0.7 to 1.1 and arm span to height ratios were above 1.0 (1.1 to 1.3$)$. Reduced trunk size to lower segment ratios suggested short trunks and increased arm span to height ratios were consistent with height deficit due to short trunk and lower limb abnormalities. Obligate carriers (IV:2, IV:5 and V:6) had normal heights 155 to $164 \mathrm{~cm}$ with -0.8 to $-2.0 \mathrm{SD}$ and no skeletal deformities were observed.

\section{Molecular genetic diagnosis}


Filtration of whole exome sequencing based on allele frequencies and homozygosity identified forty-eight variants for further analysis (Table S1). Since a number of these variants were also present in the homozygous condition in the public databases (gnomAD; https://gnomad.broadinstitute.org/ and ExAC; (http://exac.broadinstitute.org/), eliminating these reduced the list to seventeen variants (Table S1). Further scrutiny removed variants which were predicted to be benign by multiple software or the affected amino acids were not conserved in evolution. Finally, a MATN3 nonsynonymous variant c.542G>A, p.(Arg181Gln) (rs778152107), (Figure 2A), located in exon 2, remained which segregated with the disease phenotype. Sanger sequencing revealed that the variant was homozygous in all affected members and heterozygous in the unaffected individual in the pedigree (Figures 1A and 2A) while DNA from unaffected individuals IV:1, IV:3, IV:9 and IV:10 were not available for analyses. The variant was not observed in 100 normal control chromosomes and was absent from South Asian population in the public databases. Rare carriers of the variant were present in the public databases with an overall allele frequency of 0.000008 (TOPMED) 0.000012 (gnomAD_exomes) and 0.000017 (ExAC), with no individuals homozygous for the variant. The variant was predicted to be damaging by all five software used; a SIFT score of 0.001 and Polyphen2 HDIV score of 1 were obtained which support the pathogenicity of the variant. However, the interpretation by ACMGAMP guidelines for the variant was "uncertain significance" (InterVar automated) but by adding the parameters that the variant segregates with phenotype and that the observed phenotype is consistent with the known disorder for the gene, it was predicted to be "likely pathogenic" (PM1, PM2, PP1, PP3, PP4 criteria) (InterVar adjusted).The variant has been submitted to the public database LOVD with variant ID \# 0000600857.

\section{Protein structure analyses}


Computational three dimensional analyses revealed no difference in structure of the MATN3 protein with the variant in comparison to that of the wild-type protein (Figures 2B).

\section{Discussion}

MATN3 plays an important role in assembling networks with other proteins and stability of cells in extracellular matrix of cartilaginous tissues. The Von Willebrand factor type A domain of MATN3 is encoded by exon 2. It has about 200 amino acids. This domain functions in bridging MATN3 with different proteins and facilitates oligomerization, filamentous network formation, cell attachment and scattering (Whittaker \& Hynes, 2002; Mabuchi et al., 2004). The amino acid Arg181, affected by the variant identified in this study, is conserved in all vertebrate species (Figure 2C). Although, it was hypothesized that the variant may affect the structure of MATN3, no difference between computational three dimensional structures of wild-type and mutant protein were predicted (Figures 2B). Therefore, it is postulated that the variant may affect the oligomerization or binding properties of MATN3, which remains to be determined experimentally.

Variants of MATN3 are associated with two allelic disorders. Multiple epimetaphyseal dysplasia (MED; OMIM\# 607078) is caused by heterozygous variants, while spondylo-epimetaphyseal dysplasia MATN3 type occurs due to homozygous variants of MATN3. Most of these variants causing both disorders are located in exon 2 which encodes von Willebrand factor A domain (Mostert et al., 2003). This is a strong indication of its vital role in MATN3 function (Mabuchi et al., 2004). About twenty-three heterozygous dominantly inherited pathogenic variants in MATN3 have been reported to cause Multiple epimetaphyseal dysplasia MED (Chapman et al., 2001; 
155

156

157

158

159

160

161

162

163

164

165

166

167

168

169

170

171

172

173

174

175

176

177

Mostert et al., 2003; Stefansson et al., 2003; Borochowitz et al., 2004; Jackson et al., 2004;

Mabuchi et al., 2004; Cotterill et al., 2005; Maeda et al., 2005; Kim et al., 2011; Jackson et al., 2012b). These variants cause phenotypes of mild short stature (SD 0 to -2.5 ) and bowed legs along with bilateral coxa vara, irregular acetabulum, knees with genu valgum and other ossification deformities of femur (Mabuchi et al., 2004; Jackson et al., 2004; Mäkitie et al., 2004; Bonafé et al., 2014).

In contrast to multiple epimetaphyseal dysplasia, only three variants in MATN3 have been associated with a recessive mode of inheritance of Spondylo-epimetaphyseal dysplasia in Arabian, Japanese and Indian families (Borochowitz et al., 2004; Jackson et al., 2004;

Shyamasundar et al., 2019). The missense variants c.973T $>$ A p.(Cys304Ser) and c.359C $>$ T p.(Thr120Met) were homozygous in affected individuals from Arabia and India, respectively (Borochowitz et al., 2004; Shyamasundar et al., 2019). The previously reported c.359 C>T p.(Thr120Met) variant was identified with c. $908 \mathrm{C}>\mathrm{T}$ p.(Thr393Met) variant in compound heterozygosity in a Japanese family (Jackson et al., 2004). Due to lack of skeletal phenotypes in obligate carriers of recessively inherited mutations, it has been postulated that these variants of MATN3 cause loss of function, while dominantly inherited heterozygous variants act by altering protein function in a dominant-negative way (Borochowitz et al., 2004).

In conclusion, we present a novel missense variant of MATN3. This is the fourth report of an autosomal recessive Spondylo-epimetaphyseal dysplasia Martilin 3 type due to a MATN3 variant. Our findings, together with previous reports, emphasize that recessively inherited variants of MATN3 cause a phenotypically homogeneous disorder. 


\section{Conflict of interest}

Authors declare no conflict of interest.

\section{Acknowledgements}

We thank all the participants for their cooperation and Bahauddin Zakariya University Multan, Pakistan, Higher Education Commission, Pakistan and School of Biological Sciences, University of the Punjab, Pakistan for the grant.

\section{Funding Sources}

This project was partially funded by Higher Education Commission, Pakistan through its International research support initiative programme (IRSIP), research grant from Research and external linkage division of Bahauddin Zakariya University Multan, Pakistan, Higher Education Commission, Pakistan institutional grant to School of Biological Sciences and a University of the Punjab grant.

\section{Accession Number}

LOVD Variant ID: 0000600857, transcript: NM_002381.4 (MATN3)

\section{References}

Anthony, S., Munk, R., Skakun, W., \& Masini, M. 2015. Multiple epiphyseal dysplasia. JAAOSJournal of the American Academy of Orthopaedic Surgeons. 23, 164-172. 
Bell, P. A., Piróg, K. A., Fresquet, M., Thornton, D. J., Boot $\square$ Handford, R. P., \& Briggs, M. D. 2012. Loss of matrilin 1 does not exacerbate the skeletal phenotype in a mouse model of multiple epiphyseal dysplasia caused by a Matn3 V194D mutation. Arthritis \& Rheumatism. 64, 1529-1539.

Belluoccio, D., Schenker, T., Baici, A., \& Trueb, B. 1998. Characterization of human matrilin-3 (MATN3). Genomics. 53, 391-394.

Bonafé, L., Mittaz-Crettol, L., Ballhausen, D., \& Superti-Furga, A. (2014). Multiple epiphyseal dysplasia, recessive GeneReviews ${ }^{\circledR}$ [Internet]: University of Washington, Seattle

Borochowitz, Z., Scheffer, D., Adir, V., Dagoneau, N., Munnich, A., \& Cormier-Daire, V. 2004. Spondylo-epi-metaphyseal dysplasia (SEMD) matrilin 3 type: homozygote matrilin 3 mutation in a novel form of SEMD. J. Med. Genet. 41, 366-372.

Chapman, K. L., Mortier, G. R., Chapman, K., Loughlin, J., Grant, M. E., \& Briggs, M. D. 2001. Mutations in the region encoding the von Willebrand factor A domain of matrilin-3 are associated with multiple epiphyseal dysplasia. Nature genetics. 28, 393.

Cormier-Daire, V. 2008. Spondylo-epi-metaphyseal dysplasia. Pract Res Clin Rheumato. 22, 3344.

Cotterill, S. L., Jackson, G. C., Leighton, M. P., Wagener, R., Mäkitie, O., Cole, W. G., \& Briggs, M. D. 2005. Multiple epiphyseal dysplasia mutations in MATN3 cause misfolding of the A $\square$ domain and prevent secretion of mutant matrilin $\square$ 3. Hum. Mutat. $26,557-565$.

Jackson, G., Barker, F., Jakkula, E., Czarny-Ratajczak, M., Mäkitie, O., Cole, W., Wright, M., Smithson, S., Suri, M., \& Rogala, P. 2004. Missense mutations in the $\beta$ strands of the 
single A-domain of matrilin-3 result in multiple epiphyseal dysplasia. J. Med. Genet. 41, $52-59$.

Jackson, G., Mittaz $\square$ Crettol, L., Taylor, J. A., Mortier, G. R., Spranger, J., Zabel, B., Le Merrer, M., Cormier $\square$ Daire, V., Hall, C. M., \& Offiah, A. 2012a. Pseudoachondroplasia and multiple epiphyseal dysplasia: A $7 \square$ year comprehensive analysis of the known disease genes identify novel and recurrent mutations and provides an accurate assessment of their relative contribution. Human mutation. 33, 144-157.

Jackson, G., Mittaz $\square$ Crettol, L., Taylor, J. A., Mortier, G. R., Spranger, J., Zabel, B., Le Merrer, M., Cormier $\square$ Daire, V., Hall, C. M., \& Offiah, A. 2012b. Pseudoachondroplasia and multiple epiphyseal dysplasia: A 7 $\square$ year comprehensive analysis of the known disease genes identify novel and recurrent mutations and provides an accurate assessment of their relative contribution. Hum. Mutat. 33, 144-157.

Kim, O. H., Park, H., Seong, M. W., Cho, T. J., Nishimura, G., Superti $\square$ Furga, A., Unger, S., Ikegawa, S., Choi, I. H., \& Song, H. R. 2011. Revisit of multiple epiphyseal dysplasia: ethnic difference in genotypes and comparison of radiographic features linked to the COMP and MATN3 genes. Am. J. Med. Genet. A. 155, 2669-2680.

Mabuchi, A., Haga, N., Maeda, K., Nakashima, E., Manabe, N., Hiraoka, H., Kitoh, H., Kosaki, R., Nishimura, G., \& Ohashi, H. 2004. Novel and recurrent mutations clustered in the von Willebrand factor A domain of MATN3 in multiple epiphyseal dysplasia. Hum. Mutat. 24, 439-440.

Maeda, K., Nakashima, E., Horikoshi, T., Mabuchi, A., \& Ikegawa, S. 2005. Mutation in the von Willebrand factor $\square$ A domain is not a prerequisite for the MATN3 mutation in multiple epiphyseal dysplasia. Am. J. Med. Genet. A. 136, 285-286. 
Mäkitie, O., Mortier, G. R., Czarny $\square$ Ratajczak, M., Wright, M. J., Suri, M., Rogala, P., Freund, M., Jackson, G. C., Jakkula, E., \& Ala $\square$ Kokko, L. 2004. Clinical and radiographic findings in multiple epiphyseal dysplasia caused by MATN3 mutations: description of 12 patients. American Journal of Medical Genetics Part A. 125, 278-284.

Mortier, G. R., Cohn, D. H., Cormier $\square$ Daire, V., Hall, C., Krakow, D., Mundlos, S., Nishimura, G., Robertson, S., Sangiorgi, L., \& Savarirayan, R. 2019. Nosology and classification of genetic skeletal disorders: 2019 revision. American Journal of Medical Genetics Part A.

Mostert, A., Dijkstra, P., Jansen, B., Van Horn, J., De Graaf, B., Heutink, P., \& Lindhout, D. 2003. Familial multiple epiphyseal dysplasia due to a matrilin $\square 3$ mutation: further delineation of the phenotype including 40 years follow $\square$ up. Am. J. Med. Genet. A. 120, 490-497.

Shyamasundar, L., Loganathan, L., Kumar, A., Selina, A., \& Madhuri, V. 2019. MATN3 Mutation Causing Spondyloepimetaphyseal Dysplasia. Indian. J. Pediatr., 1-2.

Stefansson, S. E., Jonsson, H., Ingvarsson, T., Manolescu, I., Jonsson, H. H., Olafsdottir, G., Palsdottir, E., Stefansdottir, G., Sveinbjornsdottir, G., \& Frigge, M. L. 2003. Genomewide scan for hand osteoarthritis: a novel mutation in matrilin-3. Am. J. Hum. Genet. 72, 1448-1459.

Wagener, R., Kobbe, B., Aszódi, A., Liu, Z., Beier, D. R., \& Paulsson, M. 2000. Structure and mapping of the mouse matrilin-3 gene (Matn3), a member of a gene family containing a U12-type AT-AC intron. Mamm. Genome. 11, 85-90.

Whittaker, C. A., \& Hynes, R. O. 2002. Distribution and evolution of von Willebrand/integrin A domains: widely dispersed domains with roles in cell adhesion and elsewhere. Mol. Biol. Cell. 13, 3369-3387. 


\section{Legends}

Figure 1 Pedigree and clinical manifestation of family PK-ST-KP-08.

A. Pedigree of family PK-ST-KP-08 in which skeletal dysplasia is recessively inherited. Black symbols represent affected individuals. Genotypes for the variant c.542 G>A p.(Arg181Gln) are shown in the pedigree, below the symbols for each participant.

B. Affected individuals IV:4, V:7 and V:8 showing their bowed legs.

C. Radiographic features of knees in three patients (IV:4, V:7 and V:8). Common radiographic features in two patients are small and broad epiphyses of the knees.

D. Radiographic features of pelvis in three patients (IV:4, V:7 and V:8). Common radiographic features in two patients are short and flattened femoral neck and crescent shape femoral head.

Figure 2 Chromatogram, Clustal Omega alignment and predicted protein structure of MATN3.

A. Chromatogram for MATN3 selected region showing c.542 C>T transition. The arrow indicates the point of variant. Reverse complement sequence is shown.

B. Predicted protein structure for wild-type and mutant MATN3. (Colored images may be observed in the online issue).

C. Multiple sequence alignment of MATN3 from diverse vertebrates showing p. Arg181 (shown in bold) conservation. Stars represent the fully conserved amino acids which are identical in all species. Colons denote conservation between amino acids of similar properties. Periods show conservation between amino acids of weakly similar properties. 


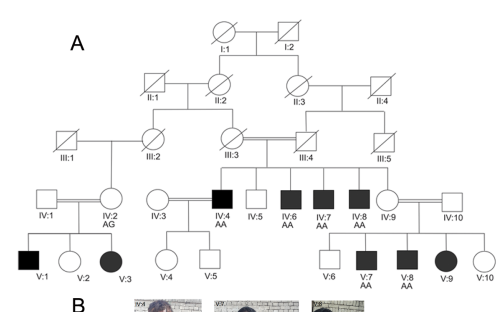

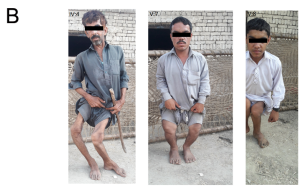

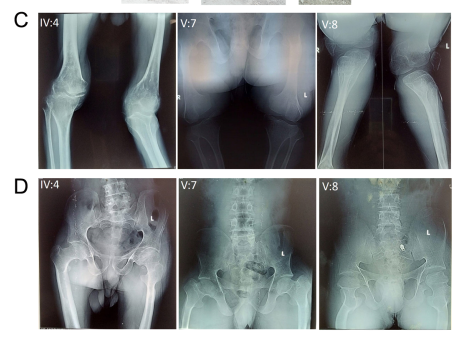


A

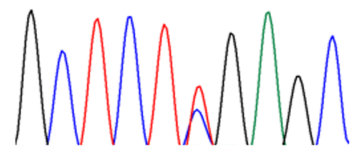

Carrier

G C T C T Y G A G C

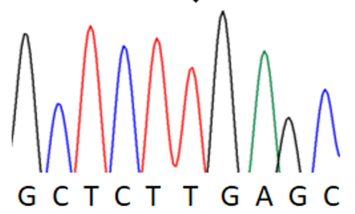

B

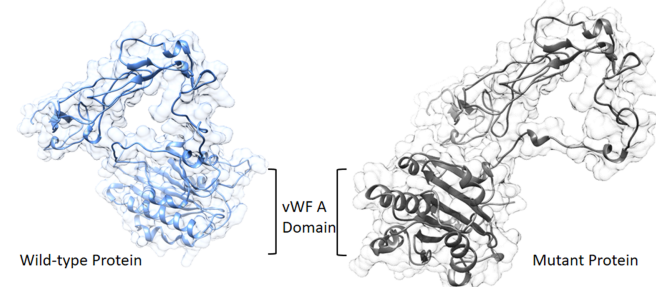

C

$\begin{array}{ll}\text { HUMAN } & \text { EAGAREPSSNIPKVAI } \\ \text { MOUSE } & \text { EAGARGPMSNIPKVAI } \\ \text { CHICK } & \text { EMGTRPANFNIPKVVI } \\ \text { ALLIGATOR } & \text { EAGARPS SLNIPKVAI } \\ \text { FROG } & \text { KAGSRRAALGIPKVAI } \\ \text { ZEBRAFISH } & \text { KSGARPKSKNISKVAI } \\ & \vdots \star \star \star\end{array}$
$: \star$ : $^{\star \star \star \star}, *$ 


\section{Highlights}

- We describe a novel homozygous variant in MATN3

- Biallelic MATN3 variant causes SEMD in six patients of a family

- The carriers had no skeletal abnormalities, ruling out dominantly inherited MED

- This is only the fourth report of SEMD due to MATN3 variant 


\section{PERMIT TO PUBLISH IDENTIFIABLE PICTURES}

My submission contains identifiable patient's images. I have collected permission from the patients and patient's guardian for the material to be published unmasked.

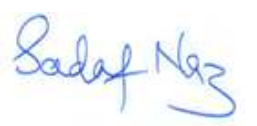

Sadaf Naz, PhD

Professor

School of Biological Sciences, University of the Punjab,

Quaid-i-Azam Campus, Lahore-54590, Pakistan

Email:naz.sbs@pu.edu.pk

Phone:92-42-99231819

Fax:92-42-99230980 
Samina Yasin ${ }^{\# 1}$, Saima Mustafa ${ }^{\# 2}$, Arzoo Ayesha $^{2}$, Muhammad Latif ${ }^{3}$, Mubashir Hassan ${ }^{4}$, Muhammad Faisal ${ }^{5}$, Outi Makitie $^{6,7}$, Furhan Iqbal ${ }^{2 \# *}$, Sadaf Naz ${ }^{1 \#}$ Institute of Pure and Applied Biology, Zoology Division. Bahauddin Zakariya University Multan 60800, Pakistan

2School of Biological Sciences, University of the Punjab, Quaid-e-Azam Campus, Lahore

3Children's Hospital, University of Helsinki, Finland

${ }^{3}$ Folkhälsan Institute of Genetics, Helsinki, Finland

4 Department of Zoology. Division of Science and Technology. University of Education Lahore, Multan Campus, Multan, Pakistan.

5 Institute of Molecular Biology and Biotechnology (IMBB). The University of Lahore, Pakistan

${ }^{6}$ Faculty of Health Studies, University of Bradford, United Kingdom

\# These authors have equal contributions

*Corresponding author

Dr. Sadaf Naz naz.sbs@pu.edu.pk

Dr. Furhan Iqbal furhan.iqbal@bzu.edu.pk

Table S1 Variants revealed after whole exome sequence analysis and sorting

\begin{tabular}{|c|c|c|c|c|c|c|c|}
\hline Chr & Start & End $\quad$ Ref & Alt & \multicolumn{3}{|c|}{ Func.refGe Gene.refG، ExonicFunc.refGene } & 1000G_ALI PROVEAN_pred \\
\hline $\operatorname{chrX}$ & $1,41 \mathrm{E}+08$ & $1,41 E+08-$ & AGAGT & exonic & MAGEC1 & frameshift insertion . & \\
\hline chr12 & 53207583 & $53207583-$ & CACCAA & exonic & KRT4 & nonframeshift insertiı. & . \\
\hline chr5 & 39202640 & $39202640 \mathrm{~A}$ & C & exonic & FYB & nonsynonymous SNV & $0,0002 \mathrm{~N}$ \\
\hline chr3 & 49162003 & 49162003 G & A & exonic & LAMB2 & nonsynonymous SNV & $0,0004 \mathrm{D}$ \\
\hline chr3 & 49136119 & $49136119 \mathrm{C}$ & G & exonic & QARS & nonsynonymous SNV & $0,0004 \mathrm{~N}$ \\
\hline chr17 & 42476569 & $42476569 \mathrm{C}$ & $\mathbf{T}$ & exonic & GPATCH8 & nonsynonymous SNV & 0,0002 \\
\hline $\operatorname{chrX}$ & 56592021 & $56592021 \mathrm{C}$ & $\mathrm{T}$ & exonic & UBQLN2 & nonsynonymous SNV . & $\mathrm{N}$ \\
\hline chr5 & 13789023 & $13789023 \mathrm{C}$ & G & exonic & DNAH5 & nonsynonymous SNV . & $\mathbf{N}$ \\
\hline
\end{tabular}




\begin{tabular}{|c|c|c|c|c|c|c|c|}
\hline chr2 & 20205753 & 20205753 C & $\mathbf{T}$ & exonic & MATN3 & nonsynonymous SNV . & D \\
\hline $\operatorname{chrX}$ & $1,53 E+08$ & $1,53 E+08 C$ & $\mathrm{~T}$ & exonic & PLXNB3 & nonsynonymous SNV . & $\mathrm{N}$ \\
\hline chr19 & 56424288 & $56424288 \mathrm{~A}$ & G & exonic & NLRP13 & nonsynonymous SNV & $0,0004 \mathrm{~N}$ \\
\hline chr3 & 47164891 & $47164891 \mathrm{C}$ & A & exonic & SETD2 & nonsynonymous SNV & $0,0002 N$ \\
\hline chr3 & 49054875 & $49054875 \mathrm{G}$ & A & exonic & DALRD3 & nonsynonymous SNV & $0,0004 \mathrm{~N}$ \\
\hline $\operatorname{chrX}$ & $1,01 E+08$ & 1,01E+08 C & $\mathrm{T}$ & exonic & ARMCX4 & nonsynonymous SNV & . \\
\hline chr14 & 55474084 & $55474084 \mathrm{~T}$ & C & exonic & WDHD1 & nonsynonymous SNV . & $\mathbf{N}$ \\
\hline chr19 & 58352848 & $58352848 \mathrm{C}$ & $\mathbf{T}$ & exonic & ZNF587B & nonsynonymous SNV . & D \\
\hline chr19 & 55085882 & $55085882 \mathrm{G}$ & A & exonic & LILRA2 & nonsynonymous SNV & $0,0004 \mathrm{~N}$ \\
\hline chr17 & 40273315 & $40273315 \mathrm{~T}$ & G & exonic & КАТ2A & nonsynonymous SNV & $0,0016 \mathrm{~N}$ \\
\hline chr17 & 40179733 & $40179733 \mathrm{C}$ & $\mathrm{T}$ & exonic & ZNF385C & nonsynonymous SNV & $0,0018 \mathrm{~N}$ \\
\hline chr13 & $1,14 \mathrm{E}+08$ & $1,14 \mathrm{E}+08 \mathrm{C}$ & A & exonic & MCF2L & nonsynonymous SNV & $0,0006 \mathrm{D}$ \\
\hline chr6 & 99323548 & $99323548 \mathrm{C}$ & $\mathbf{T}$ & exonic & FBXL4 & nonsynonymous SNV & $0,0008 N$ \\
\hline chr3 & $1,22 E+08$ & $1,22 E+08 C$ & $\mathrm{~T}$ & exonic & IQCB1 & nonsynonymous SNV & $0,001 \mathrm{~N}$ \\
\hline chr14 & 75388058 & $75388058 \mathrm{G}$ & A & exonic & RPS6KL1 & nonsynonymous SNV & $0,0014 \mathrm{D}$ \\
\hline chr5 & 36269540 & $36269540 \mathrm{G}$ & A & exonic & RANBP3L & nonsynonymous SNV . & D \\
\hline $\operatorname{chrX}$ & $1,14 \mathrm{E}+08$ & $1,14 \mathrm{E}+08 \mathrm{G}$ & A & exonic & RBMXL3 & nonsynonymous SNV & $0,0005 \mathrm{~N}$ \\
\hline chr5 & $1,78 E+08$ & $1,78 E+08 C$ & $\mathrm{~T}$ & exonic & COL23A1 & nonsynonymous SNV . & $\mathbf{N}$ \\
\hline chr17 & 18659398 & $18659398 \mathrm{C}$ & $\mathrm{T}$ & exonic & FBXW10 & nonsynonymous SNV & $0,0014 \mathrm{D}$ \\
\hline chr3 & $1,96 E+08$ & $1,96 E+08$ G & A & exonic & WDR53 & nonsynonymous SNV . & $\mathbf{N}$ \\
\hline chr3 & $1,14 \mathrm{E}+08$ & $1,14 \mathrm{E}+08 \mathrm{G}$ & A & exonic & ZNF80 & nonsynonymous SNV . & N \\
\hline $\operatorname{chrX}$ & $1,41 E+08$ & $1,41 E+08-$ & \multicolumn{2}{|c|}{ TTTTGAGG exonic } & MAGEC1 & stopgain & \\
\hline chr3 & 49940923 & $49940923 \mathrm{~A}$ & G & exonic & MST1R & synonymous SNV & 0,0004 . \\
\hline $\operatorname{chrX}$ & 30255058 & $30255058 \mathrm{G}$ & A & exonic & MAGEB3 & synonymous SNV & 0,0005 . \\
\hline chr5 & 55110843 & 55110843 A & G & exonic & DDX4 & synonymous SNV & 0,0004 . \\
\hline $\operatorname{chrX}$ & $1,18 E+08$ & $1,18 E+08 C$ & $\mathrm{~T}$ & exonic & DOCK11 & synonymous SNV & 0,0005 . \\
\hline chr5 & 36166729 & 36166729 A & G & exonic & SKP2 & synonymous SNV & \\
\hline $\operatorname{chr} X$ & 65819416 & $65819416 \mathrm{~A}$ & G & exonic & EDA2R & synonymous SNV & \\
\hline $\operatorname{chrX}$ & 70602486 & $70602486 \mathrm{~A}$ & G & exonic & TAF1 & synonymous SNV & \\
\hline $\operatorname{chrX}$ & $1,36 E+08$ & $1,36 E+08 A$ & G & exonic & ARHGEF6 & synonymous SNV & . \\
\hline chr14 & 62541899 & $62541899 \mathrm{C}$ & $\mathbf{T}$ & exonic & SYT16 & synonymous SNV & . \\
\hline chr19 & 54649507 & $54649507 \mathrm{C}$ & $\mathrm{T}$ & exonic & CNOT3 & synonymous SNV & 0,0006 . \\
\hline chr17 & 39684437 & $39684437 \mathrm{G}$ & A & exonic & KRT19 & synonymous SNV & 0,001 \\
\hline chr3 & 47088007 & $47088007 \mathrm{~A}$ & G & exonic & SETD2 & synonymous SNV & 0,0014 . \\
\hline
\end{tabular}




\begin{tabular}{|c|c|c|c|c|c|c|c|}
\hline $\operatorname{chrX}$ & $1,19 E+08$ & $1,19 E+08 C$ & $\mathrm{~T}$ & exonic & NKAP & synonymous SNV & 0,0016 \\
\hline chr18 & 33744452 & 33744452 G & $A$ & exonic & ELP2 & synonymous SNV & 0,0008 \\
\hline chr3 & 45751064 & 45751064 A & G & exonic & SACM1L & synonymous SNV & 0,0002 \\
\hline $\operatorname{chrX}$ & $1,54 \mathrm{E}+08$ & $1,54 E+08$ G & $A$ & exonic & PLXNA3 & synonymous SNV & 0,0008 \\
\hline $\operatorname{chrX}$ & 64955167 & $64955167 \mathrm{G}$ & $A$ & exonic & MSN & synonymous SNV & \\
\hline chr3 & 57438720 & $57438720 \mathrm{G}$ & $A$ & exonic & DNAH12 & unknown & 0,0006 \\
\hline chrX & 50213340 & 50213363 GGTTCTGC & & exonic & DGKK & unknown & \\
\hline
\end{tabular}

Variants remaining after removing those homozygous in controls, non-conserved and predictions of being benign are shown in bold. 